\title{
Laparoendoscopic Single-Site Surgery for Management of Gossypiboma Masquerading as an Adnexal Mass
}

\author{
Akihiro Takeda, MD, Sanae Shinone, MD, and Hiromi Nakamura, MD
}

\begin{abstract}
Background: A gossypiboma is a pseudotumor caused by a foreign-body reaction developing around inadvertently retained surgical materials in the body. Although advancements in surgical techniques and equipment has enabled successful laparoscopic management for selected cases of gossypiboma, management of gossypiboma with laparoendoscopic single-site (LESS) surgery has never been described.

Case: A 46-year-old, gravida 1, para 1 woman, who had a vaginal hysterectomy for a myoma 3 years prior, was referred due to a pelvic mass identified when she visited to her physician because of right back pain. Imaging showed a heterogeneous mass in the right adnexal region. She underwent transumbilical LESS surgery.

Results: The transumbilical LESS surgery revealed that she had an encapsulated mass with bowel adhesion in the right adnexal region adjacent to ovarian tissue. When the capsular tissue was partially penetrated by careful blunt dissection, retained surgical gauze emerged and was extracted successfully without extension of the umbilical wound. The patient's postsurgical course was uneventful.
\end{abstract}

Conclusions: A minimally invasive approach with LESS surgery could be a feasible option for management of gossypiboma in selected cases. ( J GYNECOL SURG 36:154)

Keywords: gossypiboma, magnetic resonance imaging, laparoendoscopic single-site surgery, retained surgical gauze, vaginal hysterectomy

\section{Introduction}

$\mathbf{G}$ OSSYPIBOMA - THE NOMENCLATURE of which is derived from a combination of the Latin gossypium (cotton) and the Swahili boma (place of concealment) ${ }^{1}$ - is caused by the accidental retention of surgical items, such as gauze, in the body during a surgical procedure. ${ }^{2}$

Gossypiboma is an old surgical complication but remains problematic even in the modern setting, as this complication can cause significant morbidity with a potential risk of litigation. ${ }^{3}$ Most cases of retained surgical items are discovered during the early postoperative period, when febrile morbidity, paralytic symptoms, and unusual abdominal pain manifests. $^{2,3}$ However, if gossypiboma shows a delayed presentation, remaining undetected for many years, this could pose a serious diagnostic and therapeutic challenge for the treating physician. ${ }^{1-3}$

Imaging modalities utilized to diagnose gossypiboma include plain radiography, ultrasonography, computed tomography (CT), and magnetic resonance imaging (MRI). ${ }^{3,4}$
Although such diagnostic procedures may play a substantial role in supporting a strong suspicion of gossypiboma, the obtained images alone might not usually be enough to draw a conclusion; hence, surgical intervention could be required for a final diagnosis, ${ }^{3,4}$ unless gauze with radio-opaque materials is the culprit.

The traditional approach to diagnosing gossypiboma is exploratory laparotomy, followed by the removal after making a correct diagnosis. ${ }^{2,3}$ With recent advances in surgical equipment and techniques, a minimally invasive approach utilizing laparoscopy has proven to be a feasible option for diagnosing and treating gossypiboma in select cases. ${ }^{3-5}$

Transumbilical laparoendoscopic single-site (LESS) surgery is now being increasingly used in various kinds of gynecologic surgeries. ${ }^{6,7}$ However, the feasibility of this minimally invasive approach for the diagnosis and management of gossypiboma has never been reported.

This article reports on a successfully managed case of gossypiboma after vaginal hysterectomy by LESS surgery ${ }^{6,7}$ without subsequent major complications.

Department of Obstetrics and Gynecology, Gifu Prefectural Tajimi Hospital, Tajimi, Gifu, Japan. 


\section{Case}

A 46-year-old, gravida 1, para 1 woman was referred due to a pelvic mass identified on CT when she consulted her physician because of right back pain. Her disease history was significant with an uneventful vaginal hysterectomy for a myoma 3 years earlier at another institution.

At the initial visit, ultrasonography (Fig. 1A) and MRI (Fig. 1B, long arrow) showed a heterogeneous mass measuring $6 \mathrm{~cm}$ in diameter, with a capsular structure in the right adnexal region adjacent to the suspected ovarian follicular structure (Fig. 1B, arrowhead). Because the initial radiologic diagnosis strongly suggested an ovarian endometrioma, gonadotropin-releasing hormone agonist (leuprorelin 1.88; ASKA Pharmaceutical, Tokyo, Japan) was administered twice. Thereafter, she opted to undergo surgical excision of the pelvic mass because of her persistent back pain.

A 2.5-cm, vertical, umbilical skin incision was made, and an Alexis wound retractor (small size; Applied Medical, Rancho Santa Margarita, CA) was placed. Gasless LESS surgery was performed by securing the surgical view with an intra-abdominal fan retractor system (Mizuho Co., Tokyo, Japan). ${ }^{6,7}$ Standard laparoscopic instruments were utilized for surgical manipulation.

Under a laparoscopic view, using a rigid 5-mm, $30^{\circ} \mathrm{En}-$ doEYE laparoscope (Olympus, Tokyo, Japan), the encapsulated mass with bowel adhesion was identified in the right adnexal region adjacent to the ovarian tissue (Fig. 2A). When the capsular tissue was partially penetrated with careful blunt dissection, retained surgical gauze emerged along with dark bloody fluid containing pus (Fig. 2B). The gauze was extracted successfully through the umbilical port without extension of the wound length (Fig. 2C).

The cavity formed after removal of the retained gauze was washed extensively with saline, followed by a final inspection for hemostasis as well as the absence of adjacent organ injury. The umbilical surgical wound was then closed with subcutaneous suturing (Fig. 2D).

\section{Results}

The duration of surgery was 41 minutes, and the blood loss was $<10 \mathrm{~mL}$. The patient's postoperative course was uneventful, and she was discharged from the hospital 4 days after her surgery.

\section{Discussion}

Gossypiboma is an important issue that should be discussed, even in the modern medical setting, as it can cause significant embarrassment for surgeons that can eventually lead to medicolegal problems. ${ }^{2}$ Although the incidence of gossypiboma in abdominal surgeries is reported to be between 1 in $1000-1500,{ }^{8}$ estimation of the actual rate is difficult, mainly because of presumed underreporting from medicolegal aspects. ${ }^{3}$

The inadvertent retention of surgical gauze can trigger biologic responses, including an aseptic granulomatous response against foreign-body and abscess formation after an exudative reaction. ${ }^{2,3}$ The associated symptoms with gossypiboma depend upon the size and location of the retained gauze as well as the type of inflammatory response. ${ }^{2,3}$

In the early postoperative period, gossypiboma may present with pain, inflammation, or paralytic symptoms, with or without mass formation, It can remain asymptomatic for a longer period of time, producing only nonspecific symptoms, ${ }^{2,3}$ as in the current patient, who had experienced back pain for quite some time before ultimately developing chronic lump formation 3 years after a vaginal hysterectomy.

Although radiologic investigations can be used to detect heterogenous masses that form around retained surgical items easily, ${ }^{3}$ as observed in the present case, the diagnostic value of such investigations for reaching a correct diagnosis is limited, unless the retained materials have radiologic markers.

Cotton materials containing bloody components can simulate hematomas, granulomatous processes, abscess formations, cystic masses, or neoplasms. ${ }^{3}$ This, coupled with the chronic and nonspecific presentation, poses a serious diagnostic difficulty. ${ }^{9}$

Likewise, in the current case, radiologic examinations were not sufficient for reaching the correct diagnosis, as the retained gauze had no radio-opaque markers. As a result, the diagnostic radiologists in the current authors' hospital, who lacked experience with gossypiboma, initially made a diagnosis of an ovarian endometrioma due to the containment of bloody materials in the mass. After a correct diagnosis was obtained by excision with surgery, the current authors agreed that the findings in the current case resembled those described in preciously reported cases of gossypiboma.

To avoid serious medicolegal problems, preventing gossypiboma is very important. It has been shown that, in an emergency setting, sudden changes in the operative

FIG. 1. Images of diagnostic findings of gossypiboma after vaginal hysterectomy. (A) Transvaginal ultrasonographic findings. (B) Sagittal T2-weighted magnetic resonance imaging showing the gossypiboma (long arrow) adjacent to the follicular structure of the right ovary (arrowhead).
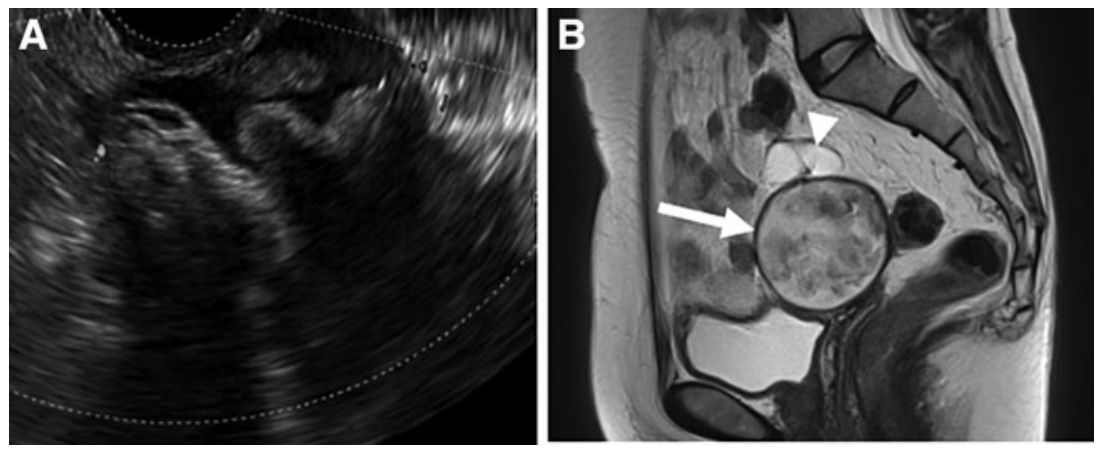

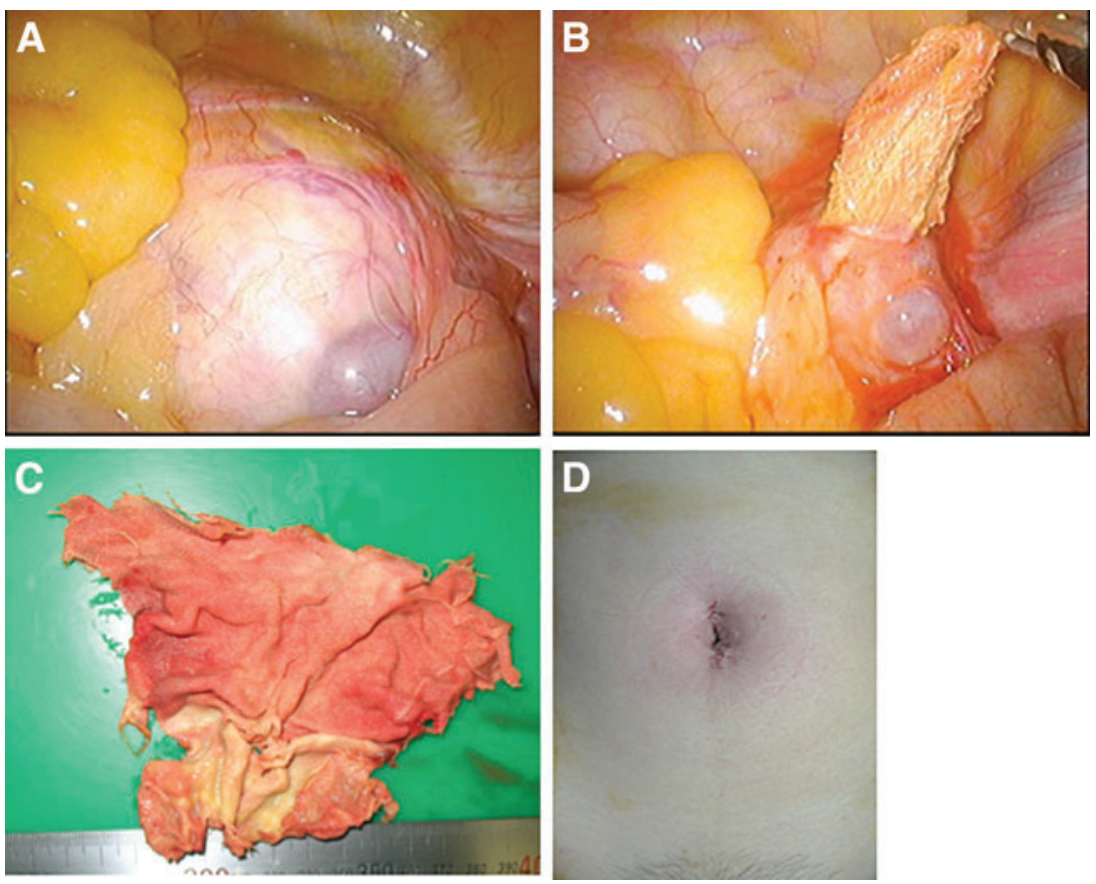

FIG. 2. (A) Laparoendoscopic singlesite view showing gossypiboma with bowel adhesion. (B) Extraction of retained gauze from encapsulated structure. (C) Extracted gauze through umbilical incision without elongation. (D) Umbilical wound after subcutaneous suturing. Color images are available online. procedure, poor communication among surgical stuff, hurried gauze counts, extended operations, a patient in unstable condition, inexperienced staff or inadequate staff numbers, and patient obesity can be potential risk factors for the development of gossypiboma. ${ }^{8}$

Therefore, to prevent the retention of foreign bodies practically, surgeons should follow the current recommendations - including the use of radio-opaque markers and simply keeping a thorough pack count during the surgical course, and perform a careful exploration of the wound and peritoneal cavity before wound closure.

At present, in the current authors' hospital, according to the recommendation of the Ministry of Health, Labour and Welfare of the Japanese Government, in addition to the use and precise count of gauze with radio-opaque markers, a routine surgical postoperative X-ray is performed to detect any missing pieces of gauze before the final closure of wounds.

However, the present patient underwent her initial procedure in a small private clinic, where gauze with such radio-opaque markers was not used. Furthermore, inspection through the vaginal canal after vaginal hysterectomy might not permit the sole investigation of the operative field in the pelvic cavity, resulting in gossypiboma forming around retained gauze.

Concerning treatment of gossypiboma, surgical removalusually through the previous operative site - can achieve a complete cure. ${ }^{3}$ However, due to the chronic nature of this disorder and the foreign-body reaction associated with various degrees of adhesion that usually form around the gossypiboma, the choice of the initial surgical approachlaparotomy or laparoscopy - may depend on surgeons' experience with laparoscopic surgery and the degree of adhesion to nearby organs (namely the bowel). ${ }^{4}$

Although a laparoscopic approach can be suitable minimally invasive option, ${ }^{3-5}$ a laparotomic approach should definitively be chosen if adhesiolysis is suspected to carry a risk of causing another iatrogenic complication, such as a bowel injury.

Another concern when considering a laparoscopic approach for extracting retained gauze is the size of the working port. In conventional multiport laparoscopic surgery, gauze cannot be exteriorized through 5-12-mm ports without extending the incisions several $\mathrm{cm}^{4}{ }^{4}$

This may be resolved, thanks to recent advances in LESS surgery, ${ }^{6,7}$ wherein laparoscopic procedures are performed through a relatively long single umbilical incision measuring from $2 \mathrm{~cm}$ to $3 \mathrm{~cm}$ in length, which can be dilated further with an Alexis wound retractor. As a result, the umbilical incisions were sufficient to exteriorize the retained gauze in the current case.

Although further accumulation of cases is needed, LESS surgery for extraction of gossypiboma may become a useful surgical option in the era of minimally invasive surgery in select cases, offering advantages for patients without any specific complications, as demonstrated in the present case.

\section{Conclusions}

Gossypiboma is a rare and avoidable but serious postoperative complication. Even in patients experiencing gossypiboma, a minimally invasive approach with LESS surgery may be considered a feasible option for minimizing abdominal scarring, thereby reducing patient dissatisfaction against iatrogenic complications.

\section{Author Disclosure Statement}

No competing financial interests exist.

\section{Funding Information}

No funding was received for this article. 


\section{References}

1. Possover M. Images in clinical medicine: Gossypiboma in the pouch of Douglas. N Engl J Med 2008;359:e9; erratum: N Engl J Med 2009;361:2004.

2. Mahran MA, Toeima E, Morris EP. The recurring problem of retained swabs and instruments. Best Pract Res Clin Obstet Gynaecol 2013;27:489.

3. Sozutek A, Colak T, Reyhan E, Turkmenoglu O, Akpınar E. Intra-abdominal gossypiboma revisited: Various clinical presentations and treatments of this potential complication. Indian J Surg 2015;77:1295.

4. Özsoy Z, Okan I, Daldal E, Dasıran MF, Angın YS, Şahin M. Laparoscopic removal of gossypiboma. Case Rep Surg 2015;2015:317240.

5. Trehan N, Singh S, Bansal Jain D. Laparoscopic management of gossypiboma. J Minim Invasive Gynecol 2019; 26:9.

6. Takeda A, Imoto S, Mori M, Nakano T, Nakamura H. Isobaric laparoendoscopic single-site assisted extracorporeal cystectomy in treatment of selected adnexal tumors: Initial experience and technique. J Minim Invasive Gynecol 2010;17:766.
7. Takeda A, Shibata M, Shinone S, Nakamura H. Emergency laparoendoscopic single-site surgery for management of iatrogenic uterine perforation: A report of 3 cases. J Gynecol Surg 2019; e-pub ahead of print:October 29; online document at: https://doi.org/10.1089/gyn.2019.0096 Accessed January 17, 2020.

8. Gawande AA, Studdert DM, Orav EJ, Brennan TA, Zinner MJ. Risk factors for retained instruments and sponges after surgery. N Engl J Med 2003;348:229.

9. Cengiz H, Kaya C, Deniztaş C, Ekin M, Ayă̆ ME, Yaşar L. Gossypiboma: After 13 years of a gynecologic proceduremasquerading as an ovarian tumor. J Obstet Gynaecol India 2014;64:81.

Address correspondence to: Akihiro Takeda, MD Department of Obstetrics and Gynecology Gifu Prefectural Tajimi Hospital 5-161 Maebata-cho, Tajimi Gifu 507-8522 Japan

E-mail: gyendoscopy@gmail.com 